\title{
La patrimonialisation de l'environnement au Biodôme de Montréal
} Gaëlle Crenn

Citer ce document / Cite this document :

Crenn Gaëlle. La patrimonialisation de l'environnement au Biodôme de Montréal. In: Culture \& Musées, n¹, 2003. Nouveaux regards sur le patrimoine (sous la direction de Jean Davallon) pp. 65-87;

doi : https://doi.org/10.3406/pumus.2003.1167

https://www.persee.fr/doc/pumus_1766-2923_2003_num_1_1_1167

Fichier pdf généré le 18/04/2018 


\begin{abstract}
As a museum ofthe environment composed of reconstituted ecosystems, Montreal 's Biodome strives to focus on the awakening of a global environmental consciousness. This is a site for making the environment a cultural heritage - where that complex thing called 'the environment' is interpreted as a legacy. Inspired by the sociology of innovation, the research conducted here aims to analyse the way in which the environment, considered in its many dimensions (scientific, political and symbolic) is established as a heritage at the Biodome. The analysis of those controversies that galvanized the Project 's beginnings allows us, first of all, to update general ideas about heritage held by various active agents - ideas that include the definition of guarantor (those who are in charge), of content (a legacy that is part of the public domain) and of the link between these two terms (cultural heritage management policy). Secondly, the idea ofwhat constitutes the environment itself is updated, through an analysis of those registers of justification (ecological, civil and 'oikological') put into work by those acting agents. Finally, the confrontation between those propositions that are under the purview of museography and the actual uses of the museum allow us to evaluate the scope of the socio-technical network that makes up the museum, as well as the impact it might acquire in the public arena. After analysis, it seems that the introduction of the environment into the museum - a process that cornes to pass by thinking hard about the very notion of heritage - also leads us to reconsider the museum 's social status as an institution for héritage building.
\end{abstract}

\title{
Résumé
}

Musée d'environnement composé d'écosystèmes reconstitués, le Biodôme de Montréal a l'ambition de " favoriser l'éveil d'une conscience environnementale planétaire ». Il est un lieu de patrimonialisation de l'environnement, où peut se lire la constitution de l'objet complexe « environnement » en patrimoine. Inspirée de la sociologie de l'innovation, la recherche rapportée ici vise à analyser la façon dont l'environnement, entendu dans ses multiples dimensions (scientifiques, politiques, symboliques), est institué en patrimoine au Biodôme. L'analyse des controverses qui émaillent la genèse du projet permet de mettre à jour dans en premier lieu les conceptions générales du patrimoine défendues par les différents acteurs, conceptions qui incluent une définition du répondant (responsable), du contenu (bien commun patrimonial), et du lien entre ces deux termes (politique de gestion du bien patrimonial). En second lieu, la constitution de l'environnement lui-même comme patrimoine est mise à jour, à travers l'analyse des registres de justification (écologique, civique et oïkologique) mobilisés par les acteurs. Enfin, la confrontation entre les propositions inscrites dans le dispositif muséographique et les usages réels du musée permet d'évaluer la taille du réseau socio-technique que constitue le musée, ainsi que le poids qu'il a pu acquérir sur la place publique. Au terme de l'analyse il apparaît que l'entrée de l'environnement au musée, processus qui s'effectue au prix d'un travail sur la notion même de patrimoine, conduit également à repenser le statut social du musée comme instance de patrimonialisation.

\section{Resumen}

Museo del medio ambiente compuesto de ecosistemas reconstituidos, el Biodôme de Montreal tiene la ambition de "favorecer el despertar de una consciencia medioam- biental planetaria ». Se trata de un lugar de patrimonialización del medio ambiente, donde puede leerse la constitución del elemento complejo " medio ambiente » en patrimonio. Inspirandose en la sociologia de la innovation, la investigación llevada a cabo, aspira a analizar la manera como el medio ambiente, interpre- tado en sus multiples dimensiones (cientificas, politicas, simbólicas), se establece como patrimonio en Biodôme. El anàlisis de las controversias que afectan la genesis del proyecto permite poner al dia en primer lugar las concepciones generales del patrimonio defendidas por los diferentes actores, concepciones que incluyen una definition del garante (responsable), del contenido (bien comùn patrimonial), y de la relación entre estos dos términos (politica de gestion del bien patrimonial). En segundo lugar, se pone al dia la constitución del propio medio ambiente como patrimonio, a través del anàlisis de los registros de justification (ecológica, cïvica y oicológica) movilizados por los actores. Por ùltimo, la confrontation entre las proposiciones inscritas en el dispositivo museogràfico y los usos reaies del museo permite evaluar la extension de la red socio-técnica que constituye el museo, asi como el peso que ha podido adquirir en la plaza pùblica. Al término del anàlisis resulta que la entrada 
del medio ambiente en el museo, proceso que se efectùa a costa de un trabajo sobre la notion misma de patrimonio, conduce asimismo a repensar el estatuto social del museo como instancia de patrimonialización. 


\title{
LA PATRIMONIALISATION DE L'ENVIRONNEMEN'T AU BIODÔME DE MONTRÉAL
}

\author{
$M$ \\ usée d'environnement \\ d'un genre nouveau, le Bio- \\ dôme de Montréal a ouvert \\ ses portes le 19 juin 1992, à \\ l'occasion du trois cent cin- \\ quantième anniversaire de \\ la fondation de la ville, et \\ accueille depuis lors près \\ d'un million de visiteurs par \\ an.
}

Situé à l'est de la cité, dans l'ancien vélodrome du complexe olympique, il expose quatre écosystèmes américains reconstitués " dans des conditions qui s'approchent le plus possible de celles du milieu naturel "(Biodôme de Montréal, 1993, p. 6). Selon ses promoteurs, le Biodôme, "réponse concrète de la société québécoise au défi environnemental ", se donne pour mission de "favoriser l'éveil d'une conscience environnementale planétaire "(Landry, 1992, p. 33).

Dans un bâtiment à l'architecture organique, les visiteurs découvrent, le long d'un chemin agrémenté de panneaux interprétatifs, la faune et la flore de La Forêt tropicale, de La Forêt laurentienne, du Saint-Laurent marin et du Monde polaire. L'ÉCOsphère, qui y introduit, présente la notion d'écosystème. Naturalia, la salle d'exploration consacrée à la notion d'adaptation, complète la visite. Les Actualités environnementales et Le Carrefour de l'environnement, où sont proposés des exemples d'intervention, ainsi qu'un centre de documentation, L'Écolothèque, incitent à l'action le visiteur interpellé en tant que citoyen responsable.

Le Biodôme se veut le lieu privilégié où se réfléchisse, au double sens d'en donner un reflet et de la mettre en question, la relation, aujourd'hui menacée, entre l'homme et la nature. Il accompagne l'émergence des questions environnementales dans le débat social, issue de l'inquiétude croissante face aux effets de l'entreprise moderne d'" arraisonnement de la nature " (Bourg, 1996 , p. 55). À l'ambition clairement affirmée de jouer un rôle social nouveau de conscientisation aux enjeux environnementaux répond une approche muséographique nouvelle : la réalisation, exemplaire par son ampleur, d'un dispositif d'immersion simulée, qui " absorbe le visiteur et lui donne l'impression d'être dans un autre lieu et dans un autre temps "(Bitgood, 1990, p. 1). Le Biodôme apparaît ainsi comme une instance privilégiée de 
patrimonialisation de l'environnement, un des lieux privilégiés où se joue et peut se lire la constitution de l'environnement en patrimoine.

L'émergence de l'environnement comme nouvel objet patrimonial apparaît comme l'ultime avatar de l'extension continue de la notion de patrimoine, depuis son acception domestique originelle - l'héritage du père -, aux objets culturels (matériels et immatériels) puis aux objets naturels. La rencontre entre le patrimoine et l'environnement ne va pas pour autant de soi, et soulève plusieurs paradoxes. De plus, l'environnement se présente d'emblée comme un objet complexe, où dimensions scientifiques, politiques et esthétiques s'entremêlent. Enfin, si l'étude de l'environnement comme patrimoine pose un défi à l'analyse, du fait de la complexité de l'objet, elle s'avère d'autant plus importante qu'elle recouvre aussi, indissociablement, une interrogation sur le rôle du musée comme instance de patrimonialisation, ainsi que sur la notion même de patrimoine.

Après avoir resitué les problèmes spécifiques que soulève l'objet environnement, nous présenterons la démarche d'analyse suivie, qui s'inscrit dans le courant de la sociologie de l'innovation. Nous rendrons compte ensuite de l'élaboration discutée au Biodôme d'une certaine conception du patrimoine, et d'une certaine conception de l'environnement comme patrimoine, élaboration saisie à travers les controverses opposant les différents acteurs du projet. Nous reviendrons pour finir sur les apports de la démarche à l'analyse des musées et de la notion de patrimoine ${ }^{1}$.

\section{LE MUSÉE FACE À UN NOUVEL OBJET COMPLEXE \\ A \\ thiborder l'objet complexe environ} requiert au premier abord de distinguer l'écologie, discipline qui assure les fondements scientifiques de la connaissance de la nature, et l'écologisme, qui concerne les discours tenus sur sa gestion. Mais l'écologie, en tant que savoir, " désigne les risques et légitime les initiatives qui contribuent à la protection et à la régénération de la nature "(Balandier, 1985, p. 176). Outre les débats au sein de la communauté scientifique entourant tant le statut que l'objet de la discipline, la complexité des liens entre écologie(s) et écologisme(s) accentue les difficultés d'analyse. En effet, l'une des caractéristiques de l'écologie systémique et des sciences contemporaines " est probablement l'extraordinaire proximité, allant jusqu'à la collusion, entre deux types de complexité : complexité scientifique (savoir incomplet et débattu) et complexité politique " (Davallon, Grandmont et Schiele, 1992, p. 36). Le Biodôme, " hommage à la nature "(Bourque, 1992, p. 22), 
s'inscrit aussi dans la longue tradition picturale et jardiniste ${ }^{2}$ des représentations de la nature. L'exposition d'environnement, offrant au regard une nature paysagée, est une expression de " la topologie imaginaire des rapports de l'homme à la nature " (Davallon, Grandmont et Schiele, 1992, p. 37). L'environnement, " objet qui se situe entre l'objet de science et l'objet anthropologique "(ibid., p. 38), engage ainsi à la fois une conception de la nature et une conception du rapport à la nature, y compris dans ses dimensions symboliques et imaginaires.

Selon Micoud, "le mot de patrimoine, et plus encore le qualificatif de patrimonial, utilisé pour caractériser un type donné de gestion, contient en lui-même une idée (et une norme) quant à ce rapport des hommes et des choses " (Micoud, 1996, p. 118). Le terme de sauvegarde recouvre ces trois dimensions de l'acte de patrimonialisation : sauver, au sens de " maintenir sauf " quelque chose qui est menacé, et garder, "qui ne veut pas seulement dire prendre soin, mais aussi garantir, se porter garant, répondre de "(ibid.). Ainsi, l'élection d'un objet en patrimoine engage la définition d'un répondant (un responsable), d'un contenu, et d'un lien qui unisse le répondant et le contenu. Les objets posés comme patrimoine " sont ipso facto institués comme hérités, indivis et irremplaçables, et corrélativement, les hommes qui en usent (qu'ils en parlent ou qu'ils les utilisent) se trouvent être qualifiés en tant que descendants, solidaires et comptables "(ibid.). L'objet patrimonialisé rassemble autour de lui les garants de sa sauvegarde, inscrit ceux-ci dans une succession et définit les modalités de sa protection.

L'acte de patrimonialisation engage un rapport à l'objet et un rapport au temps. L'une des difficultés pour appréhender l'environnement comme patrimoine réside dans le fait que ces deux rapports sont paradoxaux. En premier lieu, si l'on voit bien comment la notion de patrimoine peut rencontrer le paysage, "qui ne se conçoit pas sans une présence humaine - le pater du patrimoine "(Mourier, 1999, p. 1), il en irait, selon Mourier, tout autrement de la nature : "Si la nature, elle aussi, devient patrimoine, alors il s'agit d'un patrimoine pour ainsi dire involontaire - et en tout cas on peut se poser à bon droit la question du légateur : Dieu, une forme de transcendance?" (Ibid.) Comme le note Godard, "l'application de la catégorie du patrimoine à la nature représente un coup de force : le patrimoine désigne l'archétype du bien approprié. [...] Il s'oppose alors sémantiquement au sauvage, à l'inappropriable. Les êtres de la nature forment la classe d'objet la plus éloignée des caractéristiques attendues pour entrer dans la logique patrimoniale "(Godard, 1990, p. 239). En second lieu, l'environnement subvertit le rapport au temps habituel du patrimoine. Le futur antérieur est le temps du patrimoine, qui vise à " faire passer dans le futur ce qui fut "(Augé, 1992, p. 20). Or, défini en termes 
de projet, non de trésor accumulé, " l'environnement se présente sous la figure d'un patrimoine virtuel "(Davallon, Grandmont et Schiele, 1992, p. 71), dont l'objet comme les répondants ne sont plus réunis dans une chaîne de transmission rattachée au passé, mais projetée dans le futur. "Si l'environnement appartient aux vivants, il prend son sens de patrimoine au regard de ceux qui viendront après. Il n'est "notre" patrimoine que dans la mesure où il est reconnu comme celui des générations à venir "(Hartog, 1998, p. 15).

Par ailleurs, l'environnement interroge, plus encore peutêtre que d'autres objets, le rôle que le musée peut avoir dans la société. Traiter de l'environnement suppose non seulement de divulguer et de vulgariser un savoir scientifique, mais de conscientiser les publics aux dimensions politiques, sociales et symboliques du rapport à la nature. Dès lors, il ne s'agit plus seulement pour le musée d'être un lieu de délectation et d'éducation informelle, mais un organe de changement social, un " activiste "(Brown, 1997, p. 39), visant à modifier par sa technologie propre - l'exposition - les attitudes et les comportements des visiteurs citoyens. C'est d'ailleurs ce qui peut expliquer la constitution relativement tardive de véritables musées de l'environnement : si les musées traitant des sciences de la vie reconnaissent dès les années soixante ces préoccupations comme leurs, les thématiques environnementales, du fait des prises de position politiques qu'implique leur traitement, restent longtemps la portion congrue des musées de sciences naturelles ${ }^{3}$.

Plusieurs difficultés s'élèvent ainsi si l'on veut étudier la conception de l'environnement, conçu comme patrimoine, au Biodôme, en tenant compte à la fois des multiples dimensions de cet objet complexe et de la place du musée dans son environnement. Inspirée du courant de la sociologie de l'innovation, la démarche suivie a consisté, en inversant la perspective habituelle des sciences sociales, à considérer l'environnement non pas comme un objet constitué que l'analyse aurait pour tâche d'expliquer, mais comme le terme d'un processus de constitution, processus qui devient l'objet même de la recherche. Le courant de la sociologie de l'innovation rassemble un ensemble d'auteurs inspirés tant par la sociologie et l'anthropologie des sciences et techniques (Michel Callon, Bruno Latour, Madeleine Akrich) que par la sociologie de l'art (Antoine Hennion, Nathalie Heinich), et dont la posture théorique et méthodologique offre des perspectives nouvelles pour l'analyse du musée.

La démarche conduit à considérer le musée comme un réseau socio-technique co-construit par l'ensemble des acteurs internes et externes, y compris les publics (Latour, 1992). Le musée est vu comme le produit de l'interdépendance de groupes variés, groupes qui tentent dans le même mouvement d'établir leur identité et les rapports qui les lient aux autres 
(Akrich, 1989 ; Zolberg, 1983). L'approche de l'innovation s'attache principalement à rendre compte des stratégies d'intéressement (Callon, 1986) par lesquelles les différents acteurs - humains et non humains (Latour, 1995) - tentent de former des alliances, d'enrôler des intermédiaires, de s'en faire reconnaître comme les porte-parole légitimes. Le musée est ainsi co-construit progressivement comme réseau socio-technique, qui s'étend, se consolide et se stabilise au gré des intéressements réussis et de leur inscription durable dans des dispositifs matériels.

Cette approche, constructiviste et pragmatique, tend à réduire le pouvoir de l'analyste en sciences sociales : plutôt que d'expliquer - c'est-à-dire renvoyer à une cause générale un phénomène particulier, et dévoiler par là la vérité du réel à ses acteurs victimes d'illusion -, la sociologie de l'innovation prend au sérieux les opérations d'attribution de cause aux objets que les acleurs réalisent pour construire leur monde. Le terme générique d'accusation recouvre les stratégies d'intéressement et les opérations d'attribution de sens aux objets, opérations performatives que les acteurs réalisent constamment (Hennion, 1993). Selon le mot d'ordre de la sociologie de l'innovation, il s'agit, pour rendre compte de l'inscription du réseau, de "suivre les acteurs dans les controverses ". Celles-ci sont les noeuds privilégiés où s'observent les tensions entre les différentes définitions de la situation. L'intérêt heuristique de cette approche réside dans l'ouverture de la démarche, qui permet d'aborder sans détermination a priori la constitution d'objets complexes, en identifiant les différents registres de justification mobilisés par les acteurs (Heinich, 1996 ; Boltanski et Thévenot, 1991). Plutôt que l'explication, la démarche favorise l'explicitation des systèmes de représentation des acteurs, systèmes qui peuvent être ambivalents et contradictoires.

La démarche s'appuie enfin sur une conception renouvelée de l'usage. L'approche de la sociologie de l'innovation consiste à " sans cesse effectuer l'aller-retour entre le concepteur et l'utilisateur, entre l'utilisateur-projet du concepteur et l'utilisateur réel, entre le monde inscrit dans l'objet et le monde décrit par son déplacement "(Akrich, 1987, p. 51-52). C'est dans l'écart entre le réseau inscrit et les usages réels que se joue le sens du réseau. Il ne peut être confondu ni avec le dispositif matériel, ni avec l'ensemble des usages remplis par le dispositif : il se définit comme "le rapport produit entre ces deux termes " (ibid.). La sociologie de l'innovation renouvelle l'approche de l'usage dans une perspective dynamique : il ne s'agit pas de considérer une demande déjà existante, mais la construction dans le même mouvement d'une demande et d'un réseau (une offre). Dès lors, les publics sont considérés dès le début de l'analyse comme des acteurs à part entière. Les stratégies visant à se faire reconnaître par les publics - c'est-à-dire à devenir les 
porte-parole légitimes du public - sont un des enjeux essentiels dans la constitution du réseau (Davallon et Triquet, 1993). Les publics sont ainsi mobilisés sous la forme de publics inscrits, bien avant la confrontation avec les publics réels (Le Goaziou, 1992). Et, finalement, la taille du réseau ne peut être évaluée que dans la mesure où il est confronté aux usages réels qui en sont faits.

\section{LE PATRIMOINE EN \\ C O N T R O V ER S E S \\ L \\ 'analyse des controverses qui émaillent la} genèse du projet au Biodôme met en lumière dans un premier temps les différentes conceptions du patrimoine proposées par les acteurs.

Dans les premières formulations du projet, les concepteurs du Biodôme justifient la création d'un nouvel équipement muséologique orienté vers l'écologie et les sciences naturelles par deux raisons : d'une part, il s'agit de " participer à l'amélioration des équipements de diffusion de la culture scientifique de la ville de Montréal, afin de répondre plus adéquatement aux besoins de la collectivité "(Coplanam, 1988, p. 8) ; d'autre part, le projet entend " créer un pôle d'attraction touristique permettant de rejoindre des clientèles nombreuses et diversifiées "(ibid.). Parallèlement, une première enquête sur les publics potentiels du futur musée inscrit massivement, à la fois un public local (les résidents de Montréal et des environs) fréquentant habituellement les équipements voisins, tels que le Parc botanique, et un public touristique, québécois et international. Cette stratégie de double positionnement vise à intéresser les institutions provinciales. En effet, pour réaliser le projet, dont le budget initial atteint trente millions de dollars, les concepteurs issus de la municipalité doivent obtenir des financements provinciaux. Tout en s'attribuant la paternité du projet, ils présentent le futur musée comme une opportunité pour Québec de rentabiliser un équipement dont la vacance est coûteuse ${ }^{4}$. Ils font entendre également qu'un tel équipement serait un moyen de favoriser la reconnaissance internationale de la province, que Montréal, à la fois municipalité et métropole du Québec, continuerait de promouvoir avec le Biodôme, après avoir accueilli l'Expo 67 et les Jeux olympiques de 1976.

Alors que les acteurs visent à établir le musée comme instance légitime de patrimonialisation, le réseau se déploie ainsi initialement dans une tension entre l'inscription municipale et provinciale du musée. En destinant le Biodôme, substitut aux anciens équipements municipaux, à la collectivité des Montréalais, les concepteurs se situent en tant que répondants d'une politique 
patrimoniale municipale : le Biodôme, emblème d'une ville verte, répond d'un bien patrimonial propre aux Montréalais. Dans le même temps, le musée est inscrit comme musée provincial ; les concepteurs présentent alors l'environnement qui sera exposé au Biodôme comme le bien commun patrimonial de tous les Québécois, objet qui sera de plus une vitrine du Québec aux yeux des publics étrangers.

Deux premières controverses se développent localement, où de nouveaux acteurs cherchent à déplacer cette inscription. À l'automne 1989, la Fédération de cyclisme, qui s'oppose à la fermeture du vélodrome, assigne les promoteurs du Biodôme devant la cour supérieure. L'architecte du stade olympique se joint aux cyclistes pour dénoncer le non-respect de la vocation sportive du bâtiment. En novembre 1990, lors de la fermeture du Jardin zoologique, les syndicats municipaux protestent contre la condamnation des anciens équipements (le Jardin zoologique et l'Aquarium) et la mise en disponibilité d'une partie des personnels. Ils trouvent des appuis parmi les usagers montréalais qui privilégient les institutions existantes, mettant en avant leur attachement affectif à des lieux qui font partie de l'histoire de la ville. Dans ces deux controverses, les usagers critiquent une dénaturation des équipements qu'ils fréquentent. Ils remettent en cause le choix du contenu patrimonial, qui ne répond pas adéquatement à leurs besoins. En arguant de leurs usages des équipements, ils mettent en cause la légitimité des promoteurs du projet à représenter les Montréalais. Ils contestent par là l'autorité du répondant municipal. Parallèlement, ils dénoncent la destination du nouveau projet à des usages et à des usagers non montréalais : ils contestent également l'inscription provinciale du musée. Ils opposent une politique patrimoniale municipale légitime à une politique provinciale illégitime. Ainsi, les critiques de ces opposants recouvrent deux tentatives de déplacements du statut du musée, et le répondant est soumis à des contestations qui touchent les deux pôles, provincial et municipal, de la légitimité qu'il veut se donner.

L'une des controverses essentielles qui traversent l'histoire du Biodôme est celle qui oppose les concepteurs aux associations de défense des animaux (notamment Greenpeace et Sheppeard) qui s'opposent au prélèvement des spécimens au milieu naturel. L'annonce de la présence de bélugas au Biodôme entraîne, dès 1989, des contestations vigoureuses de leur part. La première stratégie des associations consiste à disqualifier le Biodôme comme répondant en déplaçant l'inscription provinciale du musée vers un niveau institutionnel supérieur. L'institution d'un répondant du patrimoine opère une nationalisation de la nature, c'est-à-dire la reconnaissance d'une espèce, d'un territoire, ou tout autre ensemble naturel comme bien commun 
inaliénable d'une communauté. Ainsi, pour les concepteurs, le béluga, emblème du Québec, est un bien inaliénable dont le Biodôme répond au nom des Québécois. Les associations tentent d'intéresser les institutions fédérales, plus rigoureuses en matière de prélèvement, et de les faire reconnaître comme répondant légitime et exclusif du bien environnemental, afin d'empêcher la présence de bélugas dans le Biodôme.

Les associations tentent également de disqualifier la légitimité du Biodôme comme répondant du patrimoine. Les procédures de disqualification passent d'abord par la mise en doute de l'authenticité de la préoccupation pour l'environnement. Les associations se mobilisent contre le projet bélugas par une campagne de lettres de protestation, orchestrée depuis les ÉtatsUnis : le Biodôme recevra deux mille lettres adressées dans un premier temps à la mairie. Les associations récusent l'inscription du musée comme institution protégeant l'intérêt général ; le Biodôme est présenté non comme une fin, mais comme un moyen pour ses responsables d'assurer leur position politique. Machine de guerre municipale en vue de l'accession de son principal promoteur, Pierre Bourques, à la mairie, il est un instrument dans un système de représentation politique perverti, ou pour le moins étranger à la grandeur environnementale.

Pour contourner ces accusations et témoigner de leur préoccupation environnementale, les concepteurs rappellent que des sommités de l'écologisme, dont l'engagement ne peut être mis en doute, participent au conseil des sages, organisme consultatif international qui a pour rôle de valider le contenu scientifique et culturel du projet. Ils cherchent par ailleurs à consolider le réseau en intéressant un ensemble d'acteurs civils engagés à leurs côtés : des collaborations diverses sont prévues avec les institutions zoologiques locales (zoos provinciaux) et internationales (zoos et aquariums nord-américains, y compris Disneyland), avec les universités québécoises, avec les organismes gouvernementaux et para-gouvernementaux ouvrant pour l'environnement. Surtout, les associations de conservation, de loisirs scientifiques et les naturalistes amateurs sont convoqués comme alliés ; le Biodôme s'inscrira dans une dynamique de promotion de l'action environnementale, il viendra " compléter les initiatives des autres mouvements et groupes de pression écologiques ", auxquels il "apportera une contribution et une collaboration de premier plan "(Coplanam, 1989, p. 12). Les concepteurs convoquent ainsi d'autres usagers de l'environnement pour consolider le réseau et valider leur légitimité de répondant de la politique environnementale.

Les associations remettent aussi en cause la représentativité des répondants. Le musée, qu'il soit municipal ou provincial, ne serait pas à apte à définir un bien commun environnemental. Les associations proposent d'autres représentants : les militants 
associatifs, usagers privilégiés de l'environnement, et porteparole d'une catégorie d'usagers de l'environnement, qui seraient mieux à même de répondre de la politique patrimoniale et de définir le bien commun de la communauté. L'action des associations témoigne de leur engagement à dénoncer la définition inadéquate du bien commun et de la politique patrimoniale par " la critique de la légalité des actions portant atteinte à l'environnement et le recours au contentieux, la remise en cause des enquêtes publiques, la dénonciation de la concertation comme simulacre. [...] Le travail qu'elles accomplissent en matière de formation et leurs activités de proposition pour l'action publique, de suivi de l'application des lois et de dénonciation de ses violations [...] témoignent de cet engagement civique de l'action écologique "(Lafaye et Thévenot, 1993, p. 510). La riposte du Biodôme face à ces accusations consiste à contester en retour la représentativité des associations et leur légitimité pour définir le bien commun : elles sont des lobbies qui suivent leurs intérêts propres et non l'intérêt général. L'argument de la nationalisation de la nature est retourné, en rappelant que les associations sont majoritairement anglophones et que la campagne est orchestrée depuis les États-Unis : les associations ne peuvent donc être légitimement représentatives ni des Québécois, ni des Canadiens, et elles ne peuvent pas légitimement répondre du patrimoine environnemental qu'il soit québécois ou canadien.

La remise en cause de la représentativité est appuyée par une critique portant sur les méthodes : la compétence des répondants pour mener une politique patrimoniale est insuffisante ; le savoir dont ils disposent et les manières de l'appliquer sont inadéquats. La stratégie consiste à disqualifier, selon un critère technique, la compétence professionnelle des responsables du Biodôme. Les associations rappellent par exemple l'échec des vétérinaires du Biodôme à sauver un petit béluga recueilli sür une plage. Deux conceptions différentes de la figure du savant (comme répondant de la politique patrimoniale) et du cadre de l'action patrimoniale (lien) s'opposent dans cette controverse. Les critiques des associations relèvent d'une conception de la connaissance marquée par la permanence de l'incertitude, et d'un rapport politique distancié entre l'État et le scientifique. La science de l'environnement est une science de la gestion des risques de la nature. Le savant, dont la légitimité découle de sa compétence scientifique, jugée par ses pairs, dessine la figure de l'expert. Soucieux des équilibres globaux fragiles, celui-ci agit avec précaution sur des ensembles qui restent toujours non maîtrisables. Le cadre de l'action est décentralisé pour permettre, avec souplesse et prudence, une action simultanément globale et locale. Les concepteurs se situent, quant à eux, plutôt du côté d'une figure saint-simonienne du 
savant. Dans un système de légitimité civique hiérarchisé, la connaissance est conçue comme la maîtrise par la démarche scientifique de l'objectivation. La connaissance " offre un tableau global pour un agent omniscient et surplombant [...] qui aurait la capacité requise pour construire et manipuler cette représentation, pour établir les mesures nécessaires à un bilan, pour mettre en évidence des effets pervers "(Lafaye et Thévenot, 1993 , p. 521-522). Son application rejoint l'idée saintsimonienne de fonder l'action collective sur la science et ses représentants, les ingénieurs. Grâce à leur connaissance des lois qui régissent les ensembles, ces derniers peuvent exercer leur action correctrice sur les déséquilibres. La légitimité de l'ingénieur est déléguée par l'État, et fait de celui-ci un technocrate. Ce mode de coordination favorise un cadre d'action centralisée et dirigée.

Les associations dénient, enfin, au musée le droit à s'instituer comme répondant unique de la définition légitime du bien commun patrimonial. La polémique autour de la représentativité du répondant engage des conceptions différentes du mode de coordination de l'action dans une société démocratique. Les concepteurs défendent plutôt un modèle hiérarchisé, dans lequel le musée est l'institution représentative unique garante du bien commun, qui instruit les citoyens des mesures à reprendre à leur compte et des attitudes auxquelles se conformer; les associations y opposent un modèle contestataire, où les citoyens rassemblés ou dispersés peuvent prendre part à la définition du bien commun et concourir à l'élaboration collective des comportements souhaitables. La politique patrimoniale est élaborée alors dans un modèle politique plus proche d'une démocratie directe que d'une démocratie représentative.

Ainsi, la conception du patrimoine inscrite au Biodôme se situe entre deux conceptions extrêmes et opposées, défendues par les concepteurs d'un côté, par les associations de l'autre, qui chacun tentent de mobiliser des alliés pour déplacer l'inscription dans leur sens. Ces controverses touchent aux trois pôles du patrimoine : le répondant, le contenu, et le lien (cf. tableau 1). Face aux concepteurs, les associations se présentent comme des répondants alternatifs, plus légitimes car plus sincères, plus concernés et plus compétents, et prônent des cadres et des modes d'action nouveaux. Cette remise en cause est d'autant plus profonde que l'extension vers le haut du niveau institutionnel (vers les institutions fédérales et au-delà vers des organismes internationaux), si elle vise à priver les institutions traditionnelles du contrôle de la politique environnementale au profit de nouvelles politiques globales/locales, témoigne aussi de la tentative de soustraire à terme la nature à tout contrôle : à la faire reconnaître comme être responsable d'elle-même. 
Les déplacements des associations témoignent d'une tentative de subversion d'une conception civique du patrimoine défendue par les concepteurs, par ce que l'on peut appeler une conception écologique du patrimoine. Dans ce nouveau cadre, la grandeur civique (l'attention portée au bien commun, la valorisation de l'intérêt général au détriment des intérêts particuliers) ferait place, dans un nouveau système de légitimité, à la cité verte, qui serait caractérisée par la grandeur écologique (Latour, 1995 b).

TABleaU 1

Les conceptions du patrimoine

Termes du patrimoine

Répondant (acteurs) :

Niveau institutionnel :

Institution répondante :

Figure du savant :

Contenu (conception

de l'environnement :
Les positions des acteurs

Concepteurs du Biodôme. Associations.

Municipal et provincial.

Fédéral (vers le niveau supérieur).

Musée, unique.

Associations, multiples (la nature elle-même).

Ingénieur, technocrate.

Expert, militant.

Environnementaliste (modéré). Radicale.

Lien (politique patrimoniale) :

Cadre de l'action :

Centralisé.

Décentralisé.

Démocratie :

Représentative.

Directe.

Système de légitimité

(cité) :

Grandeur civique.

Grandeur écologique.

Conception du patrimoine: Patrimoine civique

Patrimoine écologique

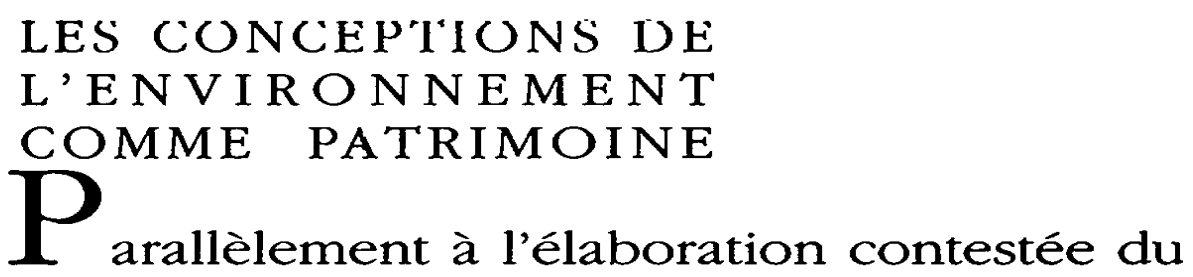
cadre du patrimoine, l'environnement est lui-même construit comme patrimoine. Trois registres de justification hétérogènes interviennent dans la définition. Le registre civique est mobilisé pour établir le répondant légitime de la nature. Le registre écologique, qui qualifie la nature comme objet de science, est convoqué pour définir la matière exposée (contenu). Le lien entre la nature et son répondant est établi selon le registre oïkologique ${ }^{6}$. Ce registre concerne, au niveau plus fondamental d'une philosophie de la nature, le rapport entre l'ordre de la 
nature et l'ordre de la société ; il définit, selon la hiérarchie établie entre ces deux ordres, le cadre d'interrelation de l'homme à la nature. Les arguments développés sur chacun des registres par les différents acteurs oscillent là encore entre des positions opposées, positions qui renvoient aux tendances, soit environnementaliste (modérée), soit radicale, de l'écologisme.

Le passage, très discuté entre muséologues et biologistes, du terme d'" habitat "à celui d'“ écosystème " pour désigner les dispositifs muséographiques, met en lumière deux conceptions opposées de la nature sur le registre écologique. Les muséologues défendent une approche naturaliste, issue de la géobotanique des voyageurs-naturalistes, où l'écologie s'attache à étudier des communautés d'espèces en interdépendance géographiquement situées. À l'opposé, les biologistes privilégient une approche biologique, qui a pour objet l'étude de systèmes ; les communautés y sont définies comme des organismes soumis à des lois mathématiques de reproduction, de succession et de transferts énergétiques (Acot, 1994 ; Drouin, 1993). Sur le registre oïkologique, une partie des formulations, qui relèvent d'une tradition humaniste, reste dans un cadre anthropocentré. L'homme garde une position éminente face à la nature dont il est responsable (l'ordre de la nature et l'ordre des sociétés sont distincts). Cette conception anthropocentrée de la nature sur le registre oïkologique est associée à une définition de la nature civique comme un être culturel, objet d'administration. La désignation du répondant de la nature est essentiellement conçue par une extension temporelle de la communauté de référence : les générations futures sont convoquées dans la communauté des répondants. Ce qu'il importe de noter ici est que, face à une nature objet, le groupe patrimonial répondant de la nature est, en tout état de cause, un groupe humain.

D'autres formulations relèvent d'une conception radicale de l'écologisme, issue de l'écologie profonde nord-américaine (deep ecology). Cette conception prend le contre-pied de l'humanisme, au profit d'un "système de valeurs biocentriques, reconnaissant à chaque espèce un droit égal à l'existence " (Bourg, 1996, p. 33). L'homme, et partant la société, sont réintégrés au sein d'un ordre naturel global (sur le registre oïkologique, l'homme est bien dans la nature). Cette conception engage un autre modèle d'extension de la communauté de référence sur le registre civique : la nature elle-même est considérée, non objet de droit, mais comme un sujet. Dès lors, le répondant de la nature devient l'ensemble des êtres naturels eux-mêmes, dont les hommes font simplement partie sans avoir de légitimité particulière à en répondre. 
TABleau 2

Cadre général de la construction de l'environnement comme patrimoine

Termes du patrimoine

Répondant (registre civique) :

Contenu (registre écologique) :

Lien (registre oïkologique) :
Les positions des acteurs

Groupe patrimonial humain ; la nature est objet.

Écologie naturaliste.

Tradition humaniste anthropocentrée : l'homme est face à la nature.
L'ensemble des êtres naturels ; la nature est le sujet. Écologie biologique symétrique.

Tradition radicale biocentrique : l'homme est dans la nature.

Au cours des controverses, les différents acteurs construisent l'environnement comme patrimoine, en établissant un cadre général où sont définis le répondant (les arguments se situent sur le registre civique), le contenu (registre écologique) et le cadre d'interrelation (registre oïkologique ${ }^{7}$ ). À travers l'étude des " disputes en justice "(Boltanski et Thévenot, 1991) entre des ordres de légitimation hétérogènes, et en tenant compte de la position adoptée sur chacun des registres, il est possible de voir comment l'environnement se construit comme patrimoine en tension entre une conception environnementaliste et une conception radicale. Dans les controverses se voient à l'ouvre les tentatives d'élaboration collective du patrimoine environnemental, sous la forme d'un nouveau sens commun, où le contrat passé entre les hommes et la nature garantirait la pérennité de celle-ci.

\section{DIMENSIONS DU RÉSEAU \\ $A$ \\ fin d'évaluer la conception du patrimoine} environnemental au Biodôme, il est nécessaire dans un second temps d'analyser les déplacements opérés par les usagers réels au cours de l'opérationalisation du musée. L'objet de patrimoine, en effet, ne devient réellement tel que s'il est reconnu par une communauté. Par cette reconnaissance, la légitimité des répondants est validée. C'est précisément dans ce processus de reconnaissance que réside la dimension collective et, de là, sociale du patrimoine : c'est en cela surtout qu'il institue un lien social. Par là aussi transparaissent la continuité et la réitération 
qui marquent l'acte de patrimonialisation (Davallon, 1992). Le patrimoine se construit à travers le cycle de définition et de reconnaissance de ces termes : il n'existe que dans les pratiques, il résulte d'un usage. Cette phase ne doit pas se comprendre comme une vérification de l'adéquation des objectifs des concepteurs aux " besoins des usagers ", que toute la dynamique de la construction progressive de l'usage récuse, mais comme " la confrontation entre usages inscrits et usages réels qui seule réalise ou irréalise "le dispositif socio-technique (Akrich, 1992, p. 52), qui seule permet d'en évaluer le sens et la portée.

L'un des déplacements majeurs observés dans les interactions $^{8}$ par rapport aux ambitions des concepteurs tient à la faible occurrence des registres civiques et oïkologiques. Ceci revient à dire que, dans les usages du musée, l'impact des activités humaines sur le milieu reste peu discuté, et les mentions d'engagement des visiteurs dans des actions en faveur de la défense de l'environnement sont faibles. À cet égard, le rôle de conscientisation aux enjeux environnementaux que voulait se donner le Biodôme ne semble que partiellement atteint. L'analyse des stratégies des acteurs et des controverses qui les ont opposés peut apporter certaines explications à ce relatif échec.

L'intégration de ces discours politiques et sociaux dans les messages proposés par le Biodôme, dans les scénarios d'animation comme dans les expositions temporaires, exige en effet d'aborder non seulement le rôle du Biodôme, mais aussi l'action d'un large ensemble d'acteurs civils intervenant à titres divers sur la nature. Ces stratégies sont délicates à mettre en cuvre car on touche à ce niveau la question de la désignation des répondants légitimes du patrimoine. Le Biodôme ne peut mentionner légitimement la politique d'autres institutions répondant du patrimoine naturel que s'il est parvenu à les intéresser suffisamment. Autrement dit, le Biodôme ne peut intégrer des propositions, concernant par exemple les politiques de gestion du vivant, que dans la mesure où ses objectifs sont suffisamment alignés avec ceux poursuivis par les autres institutions qui promeuvent des messages similaires, si les liens réels entretenus avec ces acteurs (institutions, associations, groupes) sont suffisamment robustes. Le Biodôme ne peut endosser les politiques de ces institutions que dans la mesure où il a réussi à faire admettre qu'ils ont des intérêts convergents. En particulier, pour inciter les visiteurs à l'engagement, le Biodôme doit, d'une part faire état de son propre engagement, et d'autre part témoigner de son association avec d'autres acteurs engagés.

Or, malgré les stratégies d'intéressement initialement entreprises, les associations effectives avec un réseau d'acteurs externes sont restées quasiment lettres mortes. Sur l'ensemble des acteurs civils dont le Biodôme voulait faire ses partenaires, peu 
le sont réellement devenus. Ainsi, le conseil des sages a arrêté son activité après l'ouverture du Biodôme. Les concepteurs attribuaient à l'inscription et à la reconnaissance du Biodôme au sein des institutions de recherche une importance fondamentale. Cette inscription est réalisée par la poursuite d'une politique d'accueil de chercheurs (les membres scientifiques de l'équipe initiale sont conseillers scientifiques et mènent des recherches au Biodôme) et par la coopération scientifique avec le Costa Rica et les universités montréalaises. Cependant, la diffusion des recherches et de leurs implications en termes d'action environnementale reste limitée. Organisée quatre fois par an sous la forme de soirées Nocturnes, les forums de discussion ne touchent qu'un public réduit 9 . La coopération avec le réseau des institutions muséales est active, en ce qui concerne la conception et la circulation d'expositions temporaires, mais les actions conjointes avec les sociétés naturalistes et les institutions gouvernementales sont en revanche très faibles. Surtout, les liens avec les associations de défense de la nature sont restés conflictuels : les controverses avec les associations militantes de défense des droits des animaux, dont le Biodôme n'a pu se faire un allié, pèsent toujours sur le réseau. Les oppositions initiales l'ont conduit à construire des alliances alternatives engageant d'autres institutions, mais à titre de simple caution, ce qui exclut de pouvoir témoigner d'engagements concrets.

Plus profondément encore, la fracture initiale avec les associations a contribué à réorienter l'inscription des publics du futur musée, et, de là, les messages proposés par le musée. Les associations militantes se sont en effet présentées dès le début du projet, à la fois comme des porte-parole d'usagers de l'environnement et des usagers potentiels du Biodôme. On peut comprendre qu'en échouant à intéresser ces associations militantes, les concepteurs aient dans lè même temps renoncé à considérer leurs membres comme public potentiel. Or ces publics sont les plus engagés dans les actions en faveur de l'environnement; ils défendent des conceptions plus critiques et radicales de l'environnement comme patrimoine, et accordent une importance décisive aux implications de l'intervention humaine sur la nature. Renonçant à inscrire ces publics, les concepteurs ont été conduits du même coup à minimiser les mentions des activités humaines dans la programmation du Biodôme. L'absence très frappante d'un public d'origine locale ${ }^{10}$, sensible au discours radical et sensibilisé à l'action environnementale - public pourtant attendu par les concepteurs et potentiellement important dans la région montréalaise -, confirme ce déplacement. Parallèlement, le quasi-abandon des Actualités environnementales conforte l'analyse de cette limitation du réseau.

Le sens du Biodôme en tant qu'instance de patrimonialisation de l'environnement se lit, à ce terme provisoire de son 
histoire, dans les tensions du réseau qu'il constitue sur la place publique. Nous pouvons conclure que les rares médiations des actions humaines et de l'engagement découlent de la place limitée acquise par l'institution dans un réseau d'acteurs civils engagés dans l'action en faveur de l'environnement. La robustesse insuffisante des intéressements réalisés limite l'impact du Biodôme, comme répondant légitime du patrimoine environnemental québécois et comme instance centrale de conscientisation aux enjeux politiques et sociaux de l'environnement. Paradoxalement, le Biodôme, en butte à plusieurs controverses, n'est pas un " terrain " suffisamment " contesté "(Karp, 1992, p. 6). En renonçant aux stratégies d'intéressement d'institutions actives et militantes, les concepteurs ont paradoxalement rétréci la taille du réseau, qui manque de " multiples voix "(ibid.).

\section{LES TRANSFORMATIONS \\ D U P A T R I M O I N E \\ $\mathrm{L}$}

'entrée de l'environnement au musée, objet patrimonial complexe qui institue des rapports inédits aux choses et au temps, a un impact majeur sur la notion même de patrimoine. Saisi par les mouvements environnementaux, le patrimoine est utilisé dans un sens normatif d'abord comme ensemble de règles à respecter pour sauvegarder une unité écologique, règles qui dépendent essentiellement du savoir scientifique sur l'évolution et la vie. Sont impliquées également dans la notion d'environnement comme patrimoine des procédures de gestion des entités naturelles, dans lesquelles les dimensions cognitive et juridique deviennent déterminantes, et enfin un ensemble de normes et d'attitudes collectives, soutenues par des représentations symboliques qui guident les rapports de l'homme à la nature.

L'environnement est un patrimoine à réaliser : il s'agit de définir les conditions permettant de préserver à l'avenir les entités naturelles. Du fait de ce statut particulier, le patrimoine devient affaire de restitution plutôt que de restauration : il ne s'agit pas de sauvegarder des objets de l'usure, mais de donner les conditions de restitution d'un objet, " ainsi conçue comme recréation, nouvelle vie et accomplissement de son destin " (Hartog, 1998, p.11), à édifier dans le futur. En quittant le paradigme de la restauration pour celui de la restitution, en intégrant des normes collectives et discutées, le patrimoine passe d'une problématique de la mémoire constituée, à transmettre, à la problématique d'une identité collective à définir, à refléter, à interroger. Le cas est encore ici extrême puisqu'au regard de l'altérité absolue que représente la nature, c'est l'identité de la communauté humaine dans son ensemble qui est 
concernée (voire, dans la version radicale de l'écologisme, mise en question par l'extension de la communauté des répondants aux ensembles naturels eux-mêmes). L'environnement ne se constitue comme patrimoine qu'au prix du travail qu'il effectue sur la notion même de patrimoine.

L'environnement est au coeur d'enjeux scientifiques, médiatiques, symboliques, disputés par de multiples groupes sociaux. Dès lors que le musée se donne pour mission de traiter d'un tel objet, l'exclusivité de la fonction patrimoniale se trouve mise en défaut. L'analyse du Biodôme comme réseau construit par l'interdépendance des acteurs internes et externes, y compris les publics, montre que le musée ne peut plus se considérer comme la seule instance légitime de patrimonialisation. On voit ainsi qu'avec son ambition de médiatiser non seulement la nature mais l'environnement, et de jouer un rôle social réflexif, l'intégration du musée au sein d'un réseau d'acteurs civiques devient déterminant. Les contestations des associations portent sur le contenu du patrimoine (quelles conceptions de l'environnement comme patrimoine le musée doit-il proposer) mais aussi sur le cadre du patrimoine : en contestant la représentativité du répondant, les associations tentent de subvertir le cadre du patrimoine, allant jusqu'à dénier à l'institution muséale ellemême la légitimité à répondre du patrimoine. La contestation vient également des usagers, qu'il faut considérer comme des acteurs à part entière. Des choix qu'ils opèrent dans leur parcours de visite (ou par leur choix de non-visite) dépendent les médiations effectives du patrimoine environnemental. Ces choix traduisent aussi de leur acceptation plus ou moins forte des présupposés de l'institution. En ce sens, la reconnaissance du musée comme instance légitime de patrimonialisation dépend pour une large part du bon vouloir des visiteurs. Loin d'être un récepteur passif, le visiteur a " une conscience aiguë [...] de [son] statut de membre du public lié à l'institution publique, et c'est à partir de ce statut qu'il peut se rendre disponible à la relation qui lui est proposée "(Le Marec, 1998, p. 396). Les déplacements observés traduisent aussi dans quelle mesure les visiteurs (et les non-visiteurs) adhèrent aux présupposés des messages de l'instance productrice du texte-source, dans quelle mesure ils reconnaissent la pertinence du message que le musée veut transmettre. Il nous semble à cet égard que l'un des principaux apports de la sociologie de l'innovation réside dans cette attention renouvelée portée aux publics comme acteurs du musée, qui permet de saisir de façon dynamique la relation de l'institution à son public, conçu comme visiteur et comme citoyen.

Si l'on tient compte des voies par lesquelles et son statut et son message sont contestés, il apparaît que dès lors que le musée traite de sujets sociaux, il n'est plus qu'une partie du 
sujet dont il traite. Il se trouve en concurrence avec d'autres acteurs sociaux pour définir le bien commun et les conditions de sa gestion, il se trouve en concurrence avec d'autres lieux d'information et de savoirs auxquels ont accès les visiteurs. L'analyse de la patrimonialisation de l'environnement au Biodôme permet de mettre en lumière et de témoigner de l'effritement du pouvoir régalien d'institution du patrimoine. Dès lors que le patrimoine implique la définition de normes d'action et d'attitudes collectives, le musée n'a plus ni le monopole de la violence symbolique, ni le pouvoir d'imposition absolue et unilatérale du sens. Il " n'a plus à imposer ses valeurs, mais à sauvegarder ce qui, au moment présent, est tenu comme patrimoine par les divers acteurs sociaux "(Hartog, 1998, p. 13). Le Biodôme est un cas exemplaire où s'observe face à un objet patrimonial nouveau ce phénomène de dispersion de la fonction patrimoniale coextensive à l'actualisation du patrimoine.

Cette situation nouvelle exige du musée qu'il repense les liens qu'il entretient avec les acteurs de la société civile, mais aussi avec ses visiteurs : le contrat de confiance établi entre l'institution et ses publics ne peut plus reposer exclusivement sur les garanties d'interprétation habituelles, à savoir l'authenticité des objets et la conformité du savoir scientifique (Davallon, 1998, p. 428). Plus généralement, la prise en compte positive de cette transition du patrimoine " de la temporalisation à la politisation " (Hartog, 1998, p. 10), qui donne au patrimoine un sens plus dynamique, apporte un éclairage nouveau et moins pessimiste au débat concernant la patrimonialisation généralisée des sociétés actuelles. Loin des craintes de " relégation immédiate du présent en passé obsolète "(Poche, 1998, p. 291), le patrimoine peut être considéré comme l'un des lieux où se révèlent, " labiles et comme naissants " (Latour, 1992, p. 1), de nouveaux états du lien social et de l'identité culturelle que se donnent les sociétés.

G. C. uriversilé de Nancy-II. 


\section{NOTES}

1. Cet article s'appuie sur notre recherche doctorale, Le Biodôme de Montréal ou la Nature médiatisée : patrimonialisation de l'environnement dans un dispositif d'immersion simulée (Crenn, 2001).

2. Le terme de " jardinisme " qu'affectionnait le jardiniste Jean-Claude Nicolas Forestier désigne " l'idée de pratique d'un art spécifique : celui des jardins * (Le Dantec, 1996, p. 11).

3. Nous nous permettons de renvoyer sur ce point à notre article " Les Musées d'environnement face aux discours écologistes " paru dans Quaderni (Crenn, 2000).

4. Le vélodrome est géré par la Régie des installations olympiques (RIO), organisme provincial chargé de la gestion du stade depuis les Jeux olympiques de 1976. Les coûts de maintenance sont régulièrement critiqués.

5. Directeur du Jardin botanique, Pierre Bourque est le responsable du projet. Il est élu maire de Montréal en 1994 à la tête d'une liste écologiste.

6. La racine grecque oïkos réfère à la fois au sens premier de maison, et au sens dérivé d'économie, entendu comme système organisé de relations.

7. D'autres registres (industriel, esthésique et esthétique) sont ultérieurement convoqués, qui vont conduire à établir, à partir de ce cadre général, les dimensions complémentaires de l'environnement, telles que les principes d'usages sociaux et économiques légitimes de la nature, les politiques de gestion des espaces naturels, et jusqu'à un véritable modèle de société, structurant le rapport symbolique à la nature. Pour une approche plus détaillée, nous renvoyons également à Crenn (2000).

8. L'enquête menée dans les écosystèmes du Biodôme a permis de recueillir les interactions verbales et comportementales des animateurs et des visiteurs et d'évaluer ainsi le patrimoine environnemental effectif, en tenant compte à la fois des dimensions de l'environnement convoquées et des positions adoptées (tendance environnementaliste ou radicale).

9. Associant une visite nocturne du Biodôme et une conférence scientifique, ces animations s'apparentent plutôt à une sortie mondaine pour une fraction (aisée et francophone) du public du Biodôme.

10. Une seconde enquête menée auprès des visiteurs a permis d'identifier trois profils de visiteurs caractérisés par leur origine géographique, leur perception et leur usage du musée, profils qui diffèrent tant qualitativement que quantitativement des publics initialement inscrits par les concepteurs. 


\section{RÉFÉRENCES BIBLIOGRAPHIQUES}

Acot (Pascal). 1994. Histoire de l'écologie. Paris : PUF (coll. Que saisje ?).

Akrich (Madeleine). 1987. * Comment décrire les objets techniques ? " in Tecbniques et Culture, $n^{\circ}$ 9, p. 49-64.

Akrich (Madeleine). 1989. * De la position relative des localités : systèmes électriques et réseaux socio-politiques ", p. 117-166 in Innovations et Ressources locales. Paris : Centre d'études de l'emploi. Akrich (Madeleine). 1992. " Des réseaux vidéocom aux réseaux électriques : machines, gestion, marchés ", p. 5-30 in Ces réseaux que la raison ignore / sous la direction de Bruno Latour. Paris : L'Harmattan.

Augé (Marc) (dir.). 1992. "Les Paradoxes du patrimoine ", p. 20-21 in Territoires de la mémoire: les collections du patrimoine etbnologique dans les écomusées. Thonon-les-Bains : L'Albaron ; Fédération des écomusées et des musées de société.

Balandier (Georges). 1985. Le Détour : Pouvoir et modernité. Paris : Fayard.

Biodôme de Montréal. Guide d'exploration. 1993. 88 p.

Bitgood (Stephen). 1990. "The immersion experience: Introductory remarks ". Tecbnical Report. 90(20), nov. 1990. Jacksonville (Alabama) : Center for Social Design, p. 1-2.

Boltanski (Luc), Thévenot (Laurent). 1991. De la justification : Les économies de la grandeur. Paris : Gallimard.

Bourg (Dominique). 1996. Les Scénarios de l'écologie. Paris: Hachette.

Bourque (Pierre). 1992. "Le Biodôme ". Quatre Temps : le Biodôme ou le Passage du temps, $\mathrm{n}^{\circ} 16$ (2), été 1992. Montréal : Société des Amis du Jardin botanique, p. 22-23.

Brown (Ellsworth H.). 1997. "Change of catalogue ". Museum News, nov.-déc. 1997 , p. 39-40.
Callon (Michel). 1986. " Eléments pour une sociologie de la traduction - in L'Année sociologique, $\mathrm{n}^{\circ} 36$, p. 170-208.

Coplanam. 1988. Le Biodôme de Montréal. Etude de faisabilité : rapport final. 15 oct. 68 p. et annexes.

Coplanam. 1989. Le Biodôme de Montréal. Etape plans préliminaires : rapport synthèse. 14 juillet. 63 p., annexes et planches de présentation.

Crenn (Gaëlle). 2000. * Les Musées d'environnement face aux discours écologistes "in Quaderni, $n^{\circ} 43$, hiver 2000 , p. 19-31.

Crenn (Gaëlle). 2001. Le Biodôme de Montréal ou la Nature médiatisée : patrimonialisation de l'environnement dans un dispositif d'immersion simulée. Thèse de doctorat : université du Québec à Montréal, département des Communications.

Davallon (Jean), Grandmont (Gérald), Schiele (Bernard). 1992. L'Environnement entre au musée. Lyon : Presses universitaires de Lyon / Québec : musée de la Civilisation. Davallon (Jean), Triquet (Eric). 1993. "Le "Public" : enjeu stratégique entre scientifiques et concepteurs . in Publics et Musées, $n^{\circ}$ 3, oct. 1993. Lyon : Presses universitaires de Lyon, p. 67-87.

Davallon (Jean). 1992. " Le musée est-il vraiment un média ? $\star$ in $\mathrm{Pu}$ blics et Musées, $\mathrm{n}^{\circ} 2$. Lyon : Presses universitaires de Lyon, p. 99-123.

Davallon (Jean). 1998. "Cultiver la science au musée? ", p. 397-434 in La Révolution de la muséologie des sciences / sous la direction de Bernard Schiele et Emlyn H. Koster. Lyon : Presses universitaires de Lyon / Sainte-Foy (Québec) : Éditions Multimondes.

Drouin (Jean-Marc). 1993. L'Écologie et son bistoire. Paris : Flammarion.

Godard (Olivier). 1990. " Environnement, mode de coordination et systèmes de légitimité : analyse 
de la catégorie du patrimoine naturel "in Revue économique, $\mathrm{n}^{\circ} 41$ (2), p. 215-241.

Hartog (François). 1998. "Patrimoine et Histoire : les temps du patrimoine ", p. 3-17 in Patrimoine et Société / sous la direction de Jean-Yves Andrieux. Rennes : Presses universitaires de Rennes (coll. Art et Société).

Heinich (Nathalie). 1996. "L'Art contemporain exposé aux rejets : contribution à une sociologie des valeurs " in Hermès, $n^{\circ} 20$, p. 193204.

Hennion (Antoine). 1993. La Passion musicale : une sociologie de la médiation. Paris : Métailié.

Karp (Ivan). 1992. "Introduction ", p. 1-17 in Museums and Communities: The Politics of Public Culture / sous la direction de Ivan Karp, Christine M. Kreamer et Steven D. Lavine. Washington DC : Museums and Communities / Smithsonian Institution Press.

Lafaye (Claudette), Thévenot (Laurent). 1993. "Une justification écologique? Conflits dans l'aménagement de la nature "in Revue française de sociologie, $n^{\circ} 34$, p. 495-524.

Landry (Johanne). 1992. "Une histoire d'amour " in Quatre Temps: le Biodôme ou le Passage du temps, $n^{\circ} 16$ (2), été 1992. Montréal : Société des Amis du Jardin botanique, p. 30-33.

Latour (Bruno). 1992. * Introduction *, p. 1-4 in Ces réseaux que la raison ignore / sous la direction de Bruno Latour. Paris : L'Harmattan. Latour (Bruno). 1995. La Science en action : introduction à la sociologie des sciences. Paris : Gallimard.

Latour (Bruno). 1995 b. a Moderniser ou écologiser? A la recherche de la "septième" cité "in Écologie politique, $\mathrm{n}^{\circ} 13$, printemps 1995, p. 5-27.

Le Dantec (Jean-Pierre). 1996. Jardins et Paysages : textes critiques de l'Antiquité à nos jours. Paris : Larousse.
Le Goaziou (Véronique). 1992. " Usages et Usagers : un travail de convergence ", p. 153-168 in Ces réseaux que la raison ignore/ sous la direction de Bruno Latour. Paris : L'Harmattan.

Le Marec (Joëlle). 1998. " Repenser la relation du musée à son public? ", p. 379-396 in La Révolution de la muséologie des sciences / sous la direction de Bernard Schiele et Emlyn H. Koster. Lyon : Presses universitaires de Lyon / Sainte-Foy (Québec) : Éditions Multimondes.

Micoud (André). 1996. "Musée et Patrimoine : deux types de rapport aux choses et au temps? " in Ilermès, $\mathrm{n}^{\circ}$ 20, p. 115-123.

Mourier (Pierre-François). 1999. " Au loup ! " in Les Carnets du paysage, $\mathrm{n}^{\circ} 4$, été $1999, \mathrm{p} .1$.

Poche (Bernard). 1998. " Le Patrimoine comme artifice de la déréalisation du monde social ", p. 291-307 in Patrimoine et Modernité/sous la direction de Dominique Poulot. Paris / Montréal : L'Harmattan.

Zolberg (Vera L.). 1983. "Les Musées d'art américains : des optiques contradictoires " in Sociologie du travail, $\mathrm{n}^{\circ} 4$, p. 446-457. 


\section{RÉSUMÉS}

\section{M}

usée d'environnement composé d'écosystèmes reconstitués, le Biodôme de Montréal a l'ambition de " favoriser l'éveil d'une conscience environnementale planétaire ". Il est un lieu de patrimonialisation de l'environnement, où peut se lire la constitution de l'objet complexe " environnement " en patrimoine. Inspirée de la sociologie de l'innovation, la recherche rapportée ici vise à analyser la façon dont l'environnement, entendu dans ses multiples dimensions (scientifiques, politiques, symboliques), est institué en patrimoine au Biodôme. L'analyse des controverses qui émaillent la genèse du projet permet de mettre à jour dans en premier lieu les conceptions générales du patrimoine défendues par les différents acteurs, conceptions qui incluent une définition du répondant (responsable), du contenu (bien commun patrimonial), et du lien entre ces deux termes (politique de gestion du bien patrimonial). En second lieu, la constitution de l'environnement lui-même comme patrimoine est mise à jour, à travers l'analyse des registres de justification (écologique, civique et oïkologique) mobilisés par les acteurs. Enfin, la confrontation entre les propositions inscrites dans le dispositif muséographique et les usages réels du musée permet d'évaluer la taille du réseau socio-technique que constitue le musée, ainsi que le poids qu'il a pu acquérir sur la place publique. Au terme de l'analyse il apparaît que l'entrée de l'environnement au musée, processus qui s'effectue au prix d'un travail sur la notion même de patrimoine, conduit également à repenser le statut social du musée comme instance de patrimonialisation.

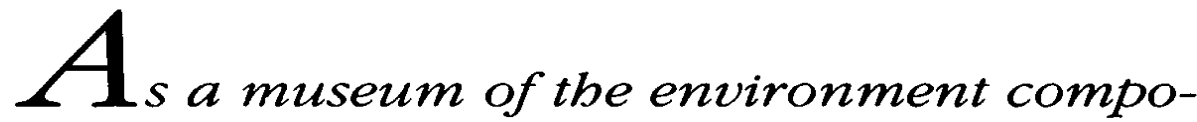
sed of reconstituted ecosystems, Montreal's Biodome strives to 'focus on the awakening of a global environmental consciousness'. This is a site for making the environment a cultural beritage - where that complex thing called 'the environment' is interpreted as a legacy. Inspired by the sociology of innovation, the research conducted bere aims to analyse the way in which the environment, considered in its many dimensions (scientific, political and symbolic) is established as a beritage at the Biodome. The analysis of those controversies that galvanized the project's beginnings allows us, first of all, to update general ideas about beritage beld by various active agents - ideas that include the definition of guarantor (those who are in charge), of content (a legacy that is part of the public domain) and of the link between these two terms (cultural beritage management policy). Secondly, the idea of what constitutes the environment itself is 
updated, through an analysis of those registers of justification (ecological, civil and 'oikological') put into work by those acting agents. Finally, the confrontation between those propositions that are under the purview of museography and the actual uses of the museum allow us to evaluate the scope of the socio-technical network that makes up the museum, as well as the impact it might acquire in the public arena. After analysis, it seems that the introduction of the environment into the museum - a process that comes to pass by thinking hard about the very notion of beritage - also leads us to reconsider the museum's social status as an institution for beritage building.

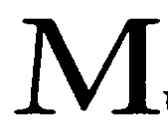

useo del medio ambiente compuesto de ecosistemas reconstituidos, el Biodôme de Montréal tiene la ambición de "favorecer el despertar de una consciencia medioambiental planetaria ". Se trata de un lugar de patrimonialización del medio ambiente, donde puede leerse la constitución del elemento complejo " medio ambiente " en patrimonio. Inspirándose en la sociología de la innovación, la investigación llevada a cabo, aspira a analizar la manera como el medio ambiente, interpretado en sus múltiples dimensiones (científicas, políticas, simbólicas), se establece como patrimonio en Biodôme. El análisis de las controversias que afectan la génesis del proyecto permite poner al día en primer lugar las concepciones generales del patrimonio defendidas por los diferentes actores, concepciones que incluyen una definición del garante (responsable), del contenido (bien común patrimonial), y de la relación entre estos dos términos (política de gestión del bien patrimonial). En segundo lugar, se pone al día la constitución del propio medio ambiente como patrimonio, a través del análisis de los registros de justificación (ecológica, cívica y oicológica) movilizados por los actores. Por último, la confrontación entre las proposiciones inscritas en el dispositivo museográfico y los usos reales del museo permite evaluar la extensión de la red socio-técnica que connstituye el museo, así como el peso que ha podido adquirir en la plaza pública. Al término del análisis resulta que la entrada del medio ambiente en el museo, proceso que se efectúa a costa de un trabajo sobre la noción misma de patrimonio, conduce asimismo a repensar el estatuto social del museo como instancia de patrimonialización. 